\title{
A New Look on the Skin Depth of the Normal Skin Effect in a Metal Submitted to a Frequency-Dependent Electromagnetic Field
}

\section{S. OlszeWSKI*}

Institute of Physical Chemistry, Polish Academy of Sciences, M. Kasprzaka 44/52, 01-224 Warsaw, Poland (Received June 4, 2012)

The theory of the skin depth in metals is re-examined by revision of the conductivity expression entering the Maxwell equations leading to the normal skin effect. In fact this conductivity formula should be improved by considering its special behaviour at low temperatures in case of the presence of the magnetic field. For very pure specimens and very low temperatures the correction of the conductivity tensor leads to the skin depth approximately proportional to the square root of the amplitude strength of the magnetic field.

DOI: 10.12693/APhysPolA.123.750

PACS: $72.30+q$

\section{Introduction}

A conventional approach to the normal skin effect in metals is based on the Maxwell equations which are [1, 2]:

$$
\begin{aligned}
& \operatorname{curl} \boldsymbol{H}=\frac{4 \pi}{c} \sigma \boldsymbol{E}, \\
& \operatorname{curl} \boldsymbol{E}=-\frac{1}{c} \frac{\partial \boldsymbol{H}}{\partial t} .
\end{aligned}
$$

The geometry applied below is such that the metal fills the half-space $x>0$ and the incident wave normal to the metal sample has $\boldsymbol{E}$ polarized along the $y$ axis and $\boldsymbol{H}$ along the $z$ axis; see [1]. Both the electric and magnetic field are regularly considered in course of the metal perturbation by a microwave.

A simplification of importance done in a former approach was the assumption that the conductivity $\sigma$ in (1), which is coupled with the current $j$ by the formula

$$
\boldsymbol{j}=\sigma \boldsymbol{E},
$$

is independent of the magnetic field. In fact, a study of the magnetoresistance effect in metals demonstrates that in the presence of the magnetic field, both the electric resistance and the electric conductivity $\sigma$, can depend essentially on the size of the magnetic field, also in their diagonal terms [3-5].

The aim of the present paper is to examine the effect of the conductivity change on the skin depth for the case when the magnetic field strength

$$
H \sim \mathrm{e}^{\mathrm{i}(\boldsymbol{k} \boldsymbol{r}-\omega t)},
$$

which oscillates in space $r$ and is modulated in time $t$ by the frequency $\omega$, is taken into account.

\section{Tensors of magnetoresistance and magnetoconductivity}

We consider here a single-band model of nearly-free electron states. The tensor describing the electric resistance in the presence of the magnetic field is in fact a superposition of two tensors. The first tensor is due to the action of the electric field alone, so it remains unin-

\footnotetext{
*e-mail: olsz@ichf.edu.pl
}

fluenced by the magnetic field. For an isotropic metal the tensor is equal to

$$
\overline{\bar{\varrho}}_{\mathrm{el}}=\frac{m}{n_{\mathrm{s}} e^{2} \tau_{\mathrm{el}}}\left(\begin{array}{lll}
1 & 0 & 0 \\
0 & 1 & 0 \\
0 & 0 & 1
\end{array}\right),
$$

$n_{\mathrm{s}}$ is the carrier concentration, $\tau_{\mathrm{el}}$ is the relaxation time in the presence of the field alone. But the second tensor depends on the direction of the magnetic field. Assuming that $\boldsymbol{H} \| \boldsymbol{z}$ we obtain [3-5]:

$$
\overline{\bar{\varrho}}_{\text {magn }}=\frac{m}{n_{\mathrm{s}} e^{2} \tau_{\text {magn }}}\left(\begin{array}{ccc}
1 & -\xi & 0 \\
\xi & 1 & 0 \\
0 & 0 & 1
\end{array}\right),
$$

where $\tau_{\text {magn }}$ is the relaxation time due to the presence of a constant field $\boldsymbol{H}$ and

$$
\xi=\frac{e H}{m c} \tau_{\text {magn }}=\omega_{H} \tau_{\text {magn }} .
$$

Here $\tau_{\text {magn }}$ is the electron circulation frequency in the field $H=H_{z}$.

A characteristic property is that $\tau_{\text {el }}$ does not practically depend on the size of $\boldsymbol{E}$, but the relaxation time $\tau_{\text {magn }}$ depends strongly on the size of $\boldsymbol{H}$. This second feature is represented by the fact that $\xi$ in (7) is a constant physically independent of $H$, so in effect $\tau_{\text {magn }}$ becomes inversely proportional to $H[3,4]$.

From a quantum-mechanical calculation of $\xi$ we obtain [3]:

$$
\xi=\frac{1}{2},
$$

whereas a semiclassical approach to $\xi$ provides us with

$$
\xi \approx \pi \text {. }
$$

The effective tensor for magnetoresistance is a sum of (5) and (6). This gives

$$
\begin{aligned}
\overline{\bar{\varrho}}_{\text {eff }} & =\overline{\bar{\varrho}}_{\text {el }}+\overline{\bar{\varrho}}_{\text {magn }}=\frac{m}{n_{\mathrm{s}} e^{2} \tau_{\text {eff }}}\left(\begin{array}{ccc}
1 & -\xi \frac{\tau_{\text {eff }}}{\tau_{\text {magn }}} & 0 \\
\xi \frac{\tau_{\text {eff }}}{\tau_{\text {magn }}} & 1 & 0 \\
0 & 0 & 1
\end{array}\right) \\
& =\frac{m}{n_{\mathrm{s}} e^{2} \tau_{\text {eff }}}\left(\begin{array}{ccc}
1 & -\xi^{\prime} & 0 \\
\xi^{\prime} & 1 & 0 \\
0 & 0 & 1
\end{array}\right) .
\end{aligned}
$$


In calculating (10) we applied the formula

$$
\frac{1}{\tau_{\text {eff }}}=\frac{1}{\tau_{\text {el }}}+\frac{1}{\tau_{\text {magn }}},
$$

which is the Matthiessen rule applied to $\tau_{\mathrm{el}}$ and $\tau_{\text {magn }}$. In the last step of (10) a substitution

$$
\xi^{\prime}=\xi \frac{\tau_{\text {eff }}}{\tau_{\text {magn }}}
$$

has been done. Formally, the tensor obtained in the final step of (10) is the reciprocal tensor of the conductivity tensor

$$
\overline{\bar{\sigma}}_{\mathrm{eff}}=\frac{n_{\mathrm{s}} e^{2} \tau_{\mathrm{eff}}}{m\left(1+\xi^{\prime 2}\right)}\left(\begin{array}{ccc}
1 & \xi^{\prime} & 0 \\
-\xi^{\prime} & 1 & 0 \\
0 & 0 & 1+\xi^{\prime 2}
\end{array}\right) ;
$$

see [2]. This is so because from (12) we have

$$
\xi^{\prime}=\frac{e H}{m c} \tau_{\text {magn }} \frac{\tau_{\text {eff }}}{\tau_{\text {magn }}}=\frac{e H}{m c} \tau_{\text {eff }} .
$$

\section{Application of the tensor (13) in the electrodynamics of metals}

The eddy current equation is obtained from a superposition of (1) and (2). This gives

$$
\text { curl curl } \boldsymbol{H}=-\nabla^{2} \boldsymbol{H}=-\frac{4 \pi \sigma}{c^{2}} \frac{\partial \boldsymbol{H}}{\partial t}
$$

from which the eddy current equation becomes

$$
k^{2} \boldsymbol{H}=\mathrm{i} \frac{4 \pi \sigma}{c^{2}} \boldsymbol{H} .
$$

A substitution of a suitable component of $\sigma^{\text {eff }}$ from (13) for $\sigma$ gives in case of the magnetic field

$$
\boldsymbol{H}=\left(0,0, H_{z}\right),
$$

where

$$
H_{z}=H_{0} \mathrm{e}^{\mathrm{i}\left(k_{z} z-\omega t\right)}
$$

the following equation for $k_{z}$ :

$$
k_{z}^{2} H_{z}=\mathrm{i} 4 \pi \sigma_{\text {eff }} \frac{\omega}{c^{2}} H_{z}=\mathrm{i} 4 \pi\left(\frac{n_{\mathrm{s}} e^{2} \tau_{\text {eff }}}{m}\right) \frac{\omega}{c^{2}} H_{z} .
$$

The expression in brackets on the right of (19) represents $\sigma_{\text {eff }}$. This provides us with a modification of a former result for $k_{z}$ which was [1]:

$$
k_{z}^{2}=\mathrm{i} 4 \pi \sigma \frac{\omega}{c^{2}}
$$

and contained $\tau_{\text {el }}$ instead of $\tau_{\text {eff }}$.

Since $k_{z}$ is a reciprocal of the spatial length of the electromagnetic wave, the next step leads to the reciprocal expression for the classical skin depth $\delta_{0}$, namely

$$
k_{z}=(1+\mathrm{i}) \frac{1}{\delta_{0}},
$$

where

$$
\delta_{0}=\left(\frac{m c^{2}}{2 \pi n_{\mathrm{s}} e^{2} \tau_{\mathrm{eff}} \omega}\right)^{\frac{1}{2}} .
$$

In principle, this $\delta_{0}$ should be real for any quantity entering the brackets in (22). However, because of the formula (11), in a very pure specimen being at very low temperature we have $\tau_{\mathrm{el}} \rightarrow \infty$ or

$$
\frac{1}{\tau_{\mathrm{el}}} \approx 0
$$

In this case the term

$$
\frac{1}{\tau_{\text {magn }}}
$$

can be a dominant component of $\frac{1}{\tau_{\text {eff }}}$ in $(11)$, so

$$
\frac{1}{\tau_{\text {eff }}} \approx \frac{1}{\tau_{\text {magn }}} .
$$

In effect, because of (7), we obtain

$$
\begin{aligned}
\delta_{0} & =\left(\frac{m c^{2}}{2 \pi n_{\mathrm{s}} e^{2} \tau_{\operatorname{magn}} \omega}\right)^{\frac{1}{2}} \cong\left(\frac{c H_{0}}{2 \pi n_{\mathrm{s}} e \xi \omega}\right)^{\frac{1}{2}} \\
& =\left(\frac{m c^{2}}{2 \pi n_{\mathrm{s}} e^{2}}\right)^{\frac{1}{2}}\left(\frac{\omega_{H}}{\omega}\right)^{\frac{1}{2}} \frac{1}{\xi^{\frac{1}{2}}} \\
& \approx 0.75 \times 10^{-5} \mathrm{~cm}\left(\frac{\omega_{H}}{\omega}\right)^{\frac{1}{2}} \frac{1}{\xi^{\frac{1}{2}}},
\end{aligned}
$$

which is a quantity proportional to $H^{\frac{1}{2}}$, or $\omega_{H}^{\frac{1}{2}}$ given in (7). The $n_{\mathrm{s}}=10^{22} \mathrm{~cm}^{-3}$ is the electron concentration assumed for a metal [6] and the constant $\xi$ is represented in (8) and (9).

In (26) we assumed that for large enough $H_{z} \sim H_{0}$ the electron circulation frequency $\omega_{H}$ in the field $H=H_{0}$ can become

$\omega_{H} \gg \omega$.

In this case a relatively small oscillation frequency $\omega$ which modifies the field $H_{0}$ into $H_{z}$ does not cancel the electron circulation effect due to $H_{0}$ alone. In a former approach [1,2] $\tau_{\text {eff }}$ in (22) was replaced by $\tau_{\text {el }}$ and no dependence of $\delta_{0}$ on $H_{0}$ was exhibited by the theory.

A special attention should be paid to the impedance $Z$ which is [1]:

$$
Z=\frac{4 \pi}{c^{2}} \frac{\omega}{k_{z}}=\frac{2 \pi \omega}{c^{2}} \delta_{0}(1-\mathrm{i}) ;
$$

see (21). Because of the formula (22) for $\delta_{0}$ we have

$$
Z=\frac{1}{c}\left(\frac{2 \pi m}{n_{\mathrm{s}} e^{2} \xi}\right)^{\frac{1}{2}}\left(\omega \omega_{H}\right)^{\frac{1}{2}}(1-\mathrm{i}) .
$$

This $Z$ has equal real and imaginary parts, a property valid also in case of an earlier formula for $Z[1,2]$.

\section{References}

[1] A.A. Abrikosov, Introduction to the Theory of Normal Metals, Academic, New York 1972.

[2] C. Kittel, Quantum Theory of Solids, 2nd ed., Wiley, New York 1976.

[3] S. Olszewski, M. Gluzinski, Z. Naturforsch. A 66 , 311 (2011).

[4] S. Olszewski, Acta Phys. Pol. A 120, 525 (2011).

[5] S. Olszewski, T. Rolinski, Z. Naturforsch. A 67, 50 (2012).

[6] C. Kittel, Introduction to Solid State Physics, 7th ed., Wiley, New York 1996. 